Volume 9 No.2, March -April 2020

International Journal of Advanced Trends in Computer Science and Engineering

Available Online at http://www.warse.org/IJATCSE/static/pdf/file/ijatcse70922020.pdf

https://doi.org/10.30534/ijatcse/2020/70922020

\title{
Tourist Spot Proposal System Using Text Mining
}

\author{
Murugan $\mathbf{G}^{1}$, Syed Musthafa $\mathrm{A}^{2}$, Abdul Jaleel $D^{3}$, Sathiya Kumar $\mathrm{C}^{4}$, Sudhakar $\mathrm{S}^{5}$ \\ ${ }^{1}$ Dept. of Computer Engineering, Vidyalankar Institute of Technology, Wadala East, Mumbai-400037 \\ Maharashtra, India, Country, gopalmurugan0@gmail.com \\ ${ }^{2}$ Dept. of IT, K.S Rangasamy College of Technology, Namakkal, India, \\ syedmusthafait@gmail.com \\ ${ }^{3}$ Dept. of CSE, Mahendra Institute of Technology, Namakkal, India, \\ abduljaleelit@gmail.com \\ ${ }^{4}$ Dept. of Computational Intelligence, Vellore Institute of Technology, Vellore \\ csathiyakumar@yahoo.com \\ ${ }^{5}$ Dept. of CSE, Sree Sakthi Engineering College, Coimbatore, Tamil Nadu, India, \\ sudhasengan@gmail.com
}

\begin{abstract}
Tourism became most popular in these decades, which the intensity of the user needs is enlarged significantly. Usually the popular travel websites give wide-ranging information such as cost details of the hotel, transportation prices, etc. But this information is not enough for the customers to get satisfied with travels in this model world. They expect more challenging information for their journey, and it should be presented on the websites, which helps to make creatively and provides more special travel plans for the users. Many more existing schemes are there on the web to improve the user for tourism. But these schemes give same and repeated information, and in many cases, the websites provide a smaller amount of secured and confidential user data and present many numbers of Ads, MNC tight-up companies oriented news for making money. In this proposed system, using the concept of text mining to recommend the best tourist spot based on budget, climate, crowd, etc. and also the data of the user are to be maintained very secure. In addition, many research activities are going on to provide more effective tourist spot for the tourists. The implementation tools are MongoDB, Angular Js, and the Spring framework is used in the development of the system. In this research designing the platform for tourists in Tamil-Nadu to include additional information about the tourist locations and also access the clear view on user review that is available in our platform helps fasten the tourist's decision-making process.
\end{abstract}

Key words: Text Mining, Decision Making, Spring Framework.

\section{INTRODUCTION}

Tourism is one of the most favorite time passes for the customer or people to spend their free time. Many guiding websites for tourists exist on the internet. However, many of the travel websites focus are based on the attention connected by making the tourist sure to enlarge their scrupulous marketplace importance and provide massive package deals. Because of this activity, they attract the user to build their journey Package extra efficient [1] [2]. These days the Recommender scheme is attractive, very prominent, and the users are attainment concerned with this because it helps to decide the most excellent package within a short span of time. In this proposed system, also included the additional functionalities to help the people or the tourist select the more efficient tourist spot based on the climatic condition of that place, crowd that is available in that spot using most advanced text mining techniques, from the tourist spot that is to be by the system the tourist can select the place according to their personal interest [3].

\subsection{Text Mining}

The Text Mining (TM) [4] concept represents a number of information enclosed within some of the substances such as articles, the newspaper, the books, some reports, good stories, the blogs, manuals, the email, and other items on the internet. The amount of text in these modern days is attractive and enormous by means of ever-growing intellectual power. The term text mining aims to recognize helpful information with no duplication as of a variety of documents through identical understanding. TM is an experimental tool so as to have the capability to identify innovative details with the intention of not too perceptible starting the collection of documents [5]. The mechanism of TM requires the generating characteristics 
within the spreadsheet arrangement, which denotes a simple and additional provisional than the open-ended mining of data scheme. It is also represented in a formless and not in a structured way. Thus it provides a very distant than the spreadsheet mechanism for data processing for predictive action. Further, this kind of data transformation as of text into a spreadsheet scheme might be extremely methodical, and this is requiring for a prearranged process to fill up in the cell of the spreadsheet [6] [7]. The text mining process is also known as Text Analytics. It represents the way to generate innovative information in huge resource collection [8].

\subsection{Importance of Text Mining}

The Text Mining mechanism increases the development of science in an excellent manner [9]. It has the latent to make easy the detection of the cure for diseases like Parkinson's and cancer.

It has been previously used to find out how the existing drugs are capable of treating in other conditions. In addition, it acts as a basis for the novelty and innovative industry. For libraries, it provides access in the direction of an increasing quantity of scientific contented, which means the researchers support for complete realize the worth of the contented holder. Thus, in turn, make sure a more exact mechanism to the researcher, as well as more during the reviews of the literature study [10] [11].

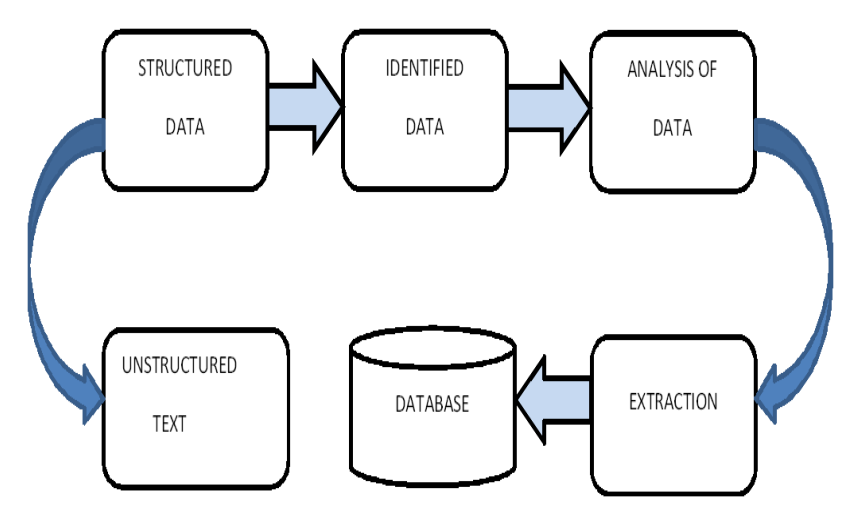

Figure 1: Text Mining

\subsection{APPLICATIONS OF TEXT MINING}

\subsubsection{Risk Management}

The essential and primary source of the failure in the business segment is short of proper or inadequate risk analysis. Adopting and making integrating the risk managing software motorized through the text mining technique like SAS TextMiner helps the businesses to stay updated by means of all latest trends in real-world marketing, which boosts their ability to alleviate the potential risks. Because the text mining approach gathers the related information among thousands of data (text) source and generates a link among extracted insights, it allows the companies to access correct information at the accurate instant, thus attracting the complete procedure of the risk management.

\subsubsection{Customer Care Service}

The Text mining methods, mainly NLP, finds mounting consequence in the customer care field. The companies are investing more money towards text analytics kind of software to improve their by and extensive customer practice by textual data accessing since various sources like customer feedback, customer surveys, and phone calls, etc. The objective of the Text analysis is to diminish the companies [12] [13] response time and helps to clear the customer grievance in a fast and efficient manner.

\section{LITERATURE REVIEW AND RELATED WORK}

In the previous scheme, the tourist web sites, observes the few existing plans afford services that go beyond a simple booking scheme's functionality. The popular method of the recommender systems might have realized the widely used in the standard filtering method. Collective modeling filtering schemes are created on the collection and the calculations of vast user information based on the historical behavior, actions, or favorites and guessing what the users preferred centered on the resemblance to the other users. The critical benefit to the collective filtering method has been that it actually doesn't trust the analyzing the content of the machine, and thus it will be gifted of exactly recommending the complicated things like movies that do not require a "thoughtful" of the element itself. For example, the theme of travel recommendation information, the primary attractive concern, is the desirability recommendation [14].

Authors Joan Borràs, AidaValls, Antonio Moreno, (2014), had given recommender schemes that are being now being functional in various domains. This paper emphasis the tourism application [15]. A complete and systematic search of this kind of smart e-Tourism endorses reported in the Journal of Artificial Intelligence [16]. It provides a comprehensive and current survey of the area, in view of the various set of interfaces, the range of algorithmic recommendation, the characteristic accessible by these schemes, and its practice of Artificial Intelligence methods. This survey also delivers some rules for the structure of tourism recommendation and the summaries of the most capable areas of the work in the field in the upcoming years [17].

The authors Shiyu Wang and Liwei Chen (2015), the previous and old way of tourism scheme's information review is imperfect, the images remain instinctive, and the consequences are not in the form of three-dimensional. To provide the solution to these difficulties, the scheme administration designs an application created on the tourist routes review. Initially, it is based on the MAP technology, 
and they attain the cross-check of space and data attributes, at that time of exploring the problem of the knapsack, concluding genetic algorithm, construction of travel route preparations added realistically and providing newest shareable maps that help for the tourists, to choose and finalize the conducts experiment simulation based on the system method [18] [19]. The results demonstrated that compared with the earliest one, this information found on the management of tourism might be faster, and similarly, its review based results provide further pictorial and three-dimensional oriented. It offers much additional suitable for the tourists through practicality.

\subsection{Analysis of the Problem}

In the previous schemes, they have numerous works that have been manually completed by tourists, and then they have not acquired the appropriate information. The tourist stayed the multiple links; nevertheless, they were not caught their endpoint. Moreover, the weather or environmental factors, transportation tools, and evolution are not mentioned. In the previous scheme, they do not recommend tourists, which holds are improved to identify, be there, and there information around the attraction has become better exposed to the tourist. Besides the recommendation scheme, it does not practice on which assistance to be chosen as a source of the web, which provides depiction to the attentive attraction grounded on additional tourist's ratings. The new presented reference scheme for the tourist like location, to proposition better user services [20].

\section{PROPOSED SYSTEM}

The new proposed scheme has a web-centered application [21] [22] and preserves a central repository to the associated information. This scheme allows the user to access the related information simply, and then it makes necessary travel preparations, booking of hotel and the navigation. The system is furnished with most excellent effectual text mining techniques to recommend the best tourist spot based on the climatic condition of that place, the crowd that is present using the feedback given by the tourist from which the users must decide the places that they needed for visiting and make bookings the travel online and lodging also filter the innards affording to the requirements of the user to mention the effective tourist spot depending upon the age of the user, the place, etc., Booking might be made using a debit card or credit card [23] — using the content scheduler to eliminate the out-dated information and providing the relevant information according to the user needs.

\subsection{Advantages of Proposed System}

- Recommends the famous tourist spots based on the recent text mining techniques on the day to day updates of the user feedback [24].

- Based on the climatic condition, the crowd on the particular spot, the system recommends the tourist spot from which the user can select the place based on their requirements.

- Maintains the confidential data of the user in a more secure manner.

- Online transactions can be done in a more secure manner.

- It provides the most attractive and straightforward GUI that makes the users find the required spot and do further process.

- Different modules do a variety of work for the betterment of the user [25].

- Maintain database schema for document modeling of data.

- Uses a specified API for performing request and response operation.

- Administration process for maintaining and update user information.

- Different travel and hotel information for the user base on their requirement

- Finally, the recommendation and user module for the user benefit.

- The below diagram explains the system architecture flow.

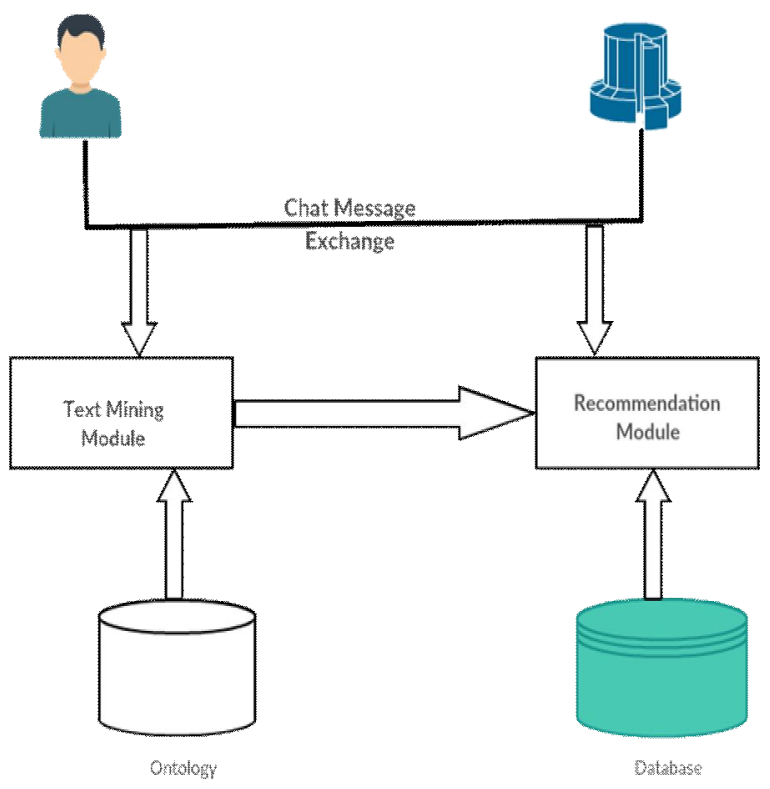

Figure 2: System Architecture

\subsection{Module Description}

The proposed work has different modules which are stated below:

\subsubsection{Database schema and model creation}

A database model is created in MongoDB using the Mongoose framework on top of it. Mongoose represents the MongoDB object modeling tool intended to work as an 
asynchronous situation. This mongoose will be the front end for MongoDB, the open-source NoSQL database been used for document-oriented modeling of data. The "collection" with "documents" in the MongoDB database.

\subsubsection{The REST API for CURD Operation}

The REST-based server API used by MongoDB has been built on the top of the HTTP server non-blocking side. REST-based API acts as a middle man and creates mapping automatically among the MongoDB's internal data storage and external graph handy HTTP resources. It is responsible for the REQUEST and RESPONSE operation between the hardware-edge and the client.

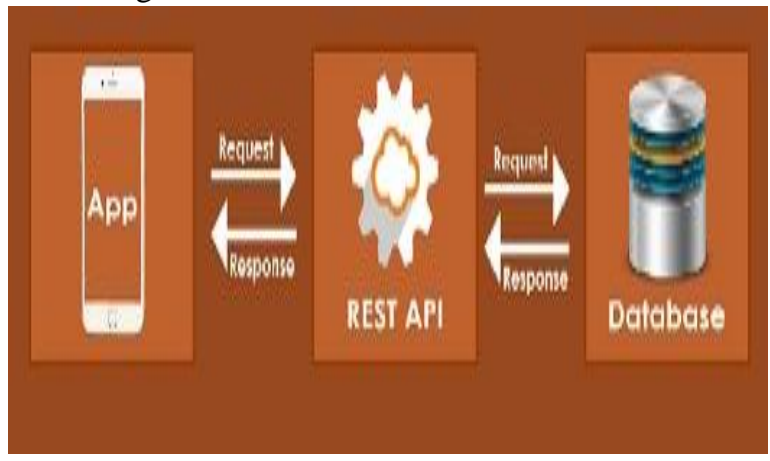

Figure 3: CURD Operation

\subsubsection{Administrator Modules}

It provides the administrator associated the functionalities such as add, modifies, and delete the details of the user. The admin can manage the details of reservation and booking information.

\subsubsection{Travel Module}

It provides various details of travel agency, from which the user can choose the suitable agency based on their convenience. It provides the shortest route from source to destination. Provides the details like source, destination, fare, reservation details, etc.

\subsubsection{Hotel Module}

Book the best hotel rooms at a low price. View the nearby hotels in the specific tourist spot. Select hotel rooms depending on the facilities that are available in the hotel like $\mathrm{a} / \mathrm{c}$, wi-fi, etc.,

\subsubsection{Recommendation Module}

They were used for recommending the appropriate tourist spot to the specific user based on the opinions, the interest that is to be given in the feedback section of the website. List the tourist spot based on the priority of the user's wish. List famous tourist spot based on the climatic conditions.

\subsubsection{User Module}

A user adds the required details for the login purpose and also views the tourist spot that is to be recommended, to book rooms, reserve the tickets for the travel.

\section{RESULTS AND DISCUSSION}

The results of the proposed work are shown and discussed below:

\subsection{Account Creation in website}

The user clicks the signup tab in the navigation bar, and the signup form appears user fill the required details and submit the form.

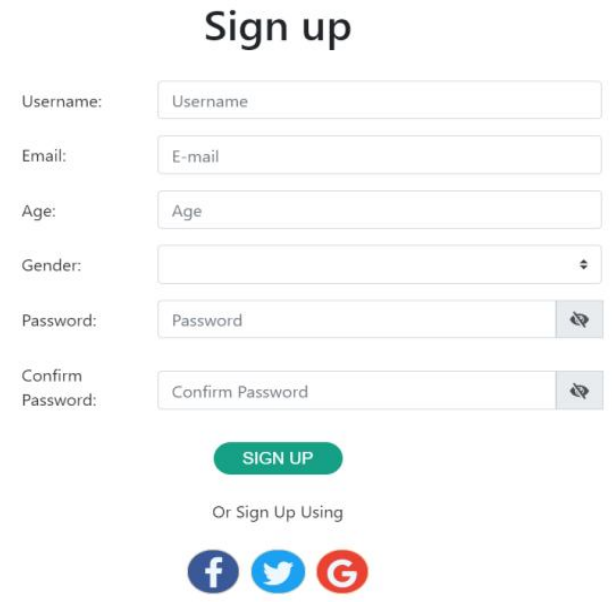

Figure 4: Sign Up Page

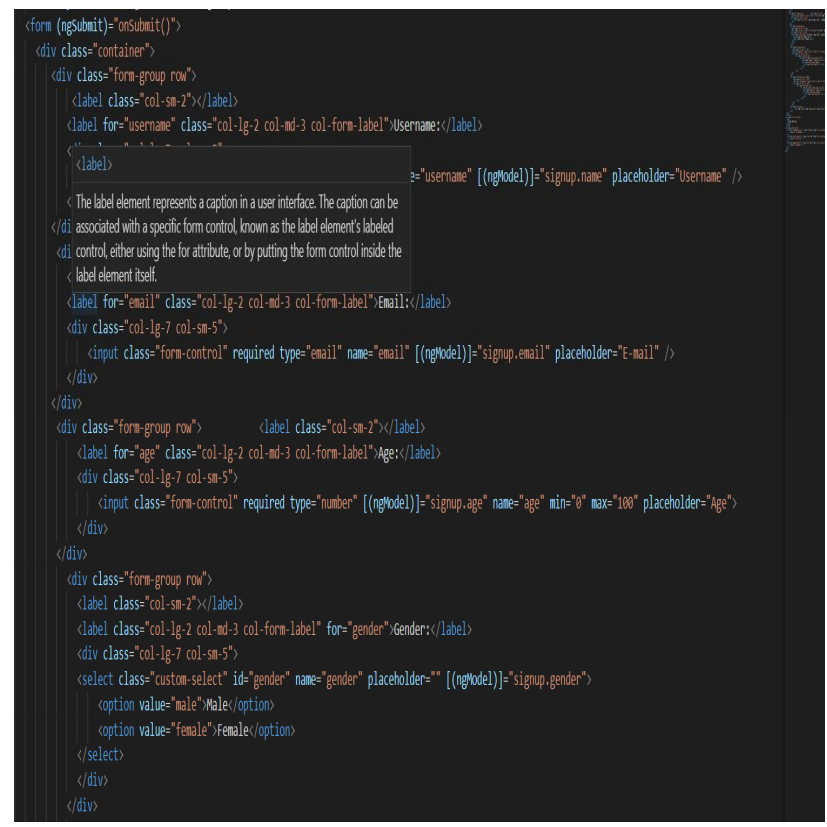

Figure 5: Code for Sign up Form 


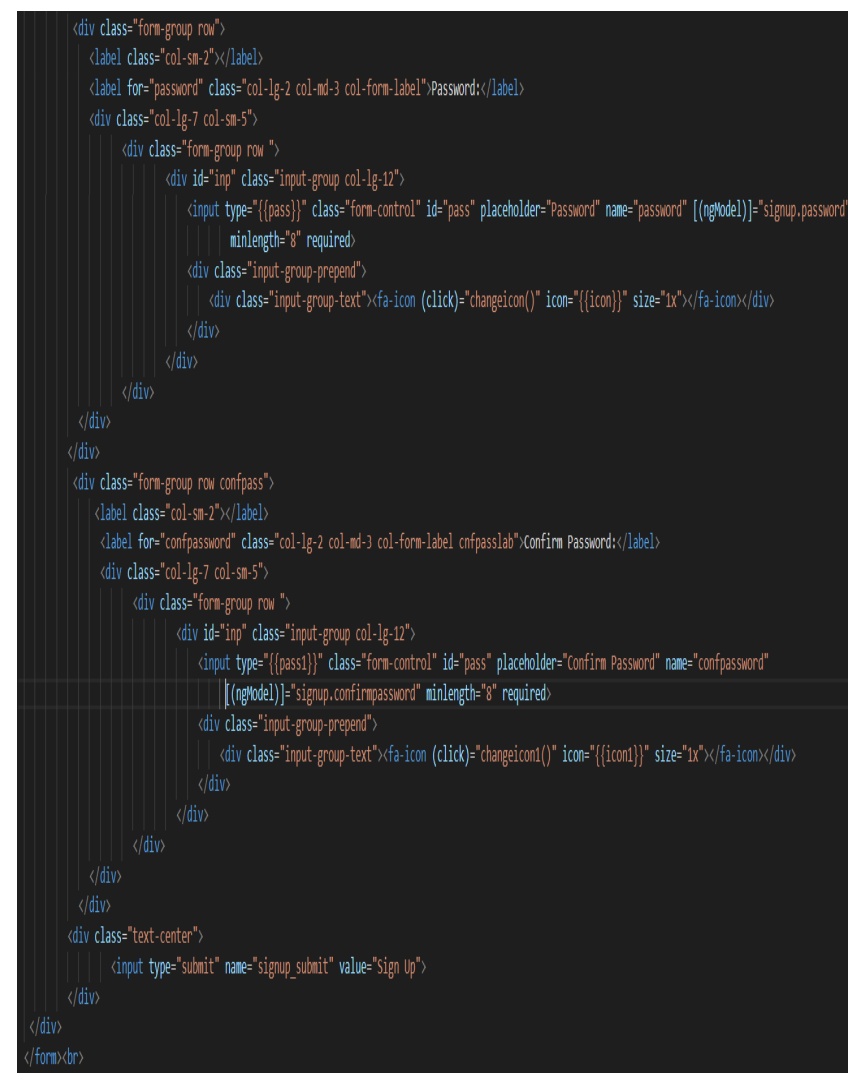

Figure 6: Coding for Sign up Form

\subsection{Account Creation in website}

Users can fill the login credentials; the details are authenticated and get redirected to the profile of the user.

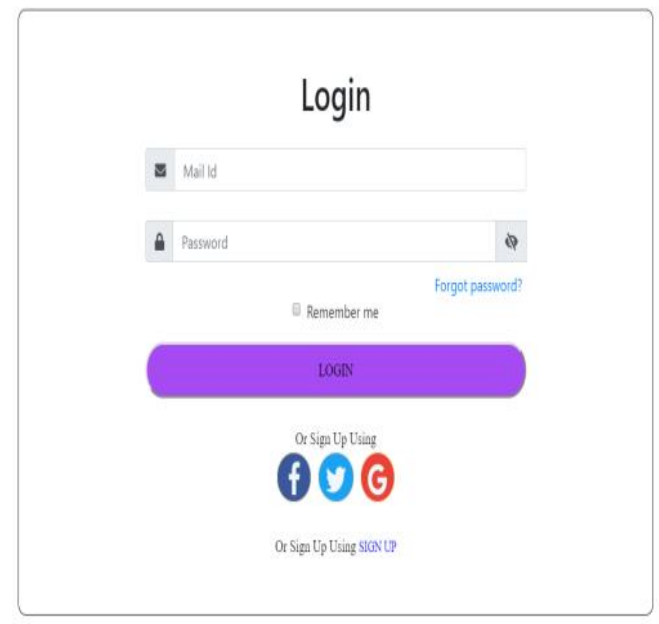

Figure 7: Login Page

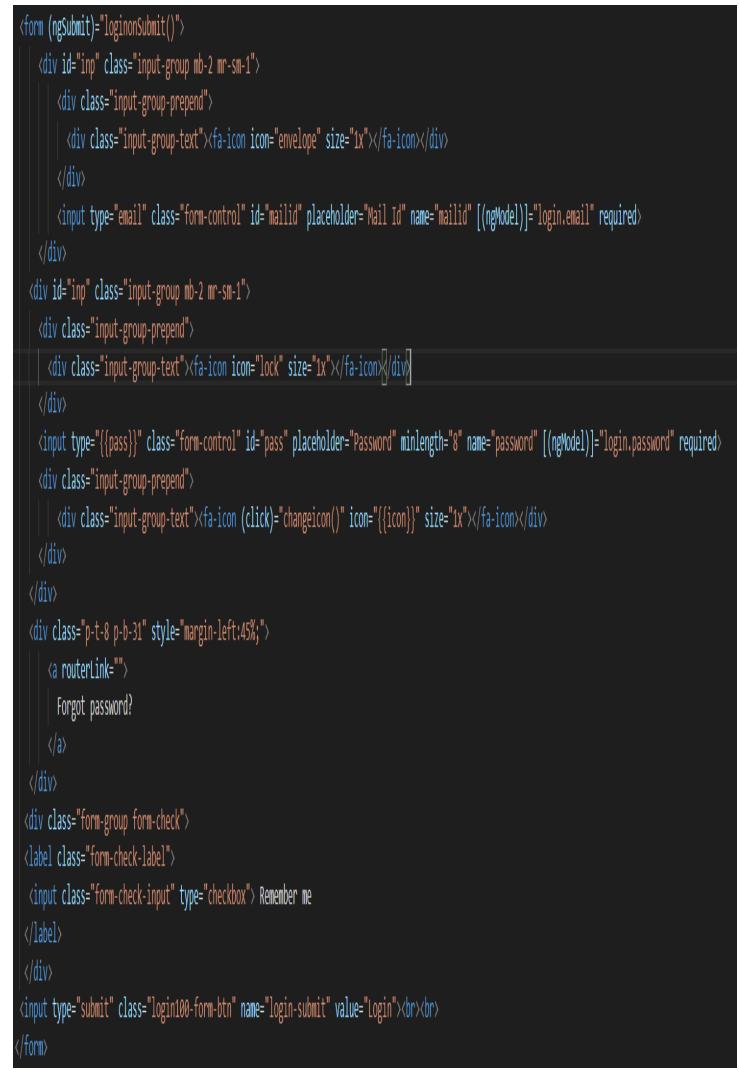

Figure 8: Code for Login Page

The above sample form and code represent the first sign up and login page of the proposed work once the user login with their valid details. The hotel recommendation and other information are displayed to the user.

\section{CONCLUSION}

Based on the analysis, the recommendation of software drives solutions for numerous problems in the Tamil-Nadu concerning the management of the product and the information affecting tourism. The tourists can get aware through entirely tourist locations in Tamil-Nadu, then the information relating to the particular sites without actually extracting the information since people or the consuming the long travel distances, to realize the locations to offer as of their choice. Through the accessibility of the web, users can access the application. Thus they permitted by the current and pertinent tourism information in Tamil-Nadu. This research plays a leading role in the tourist in making the decision, similarly by way of the source of income to the country. The system is equipped with the most efficient text mining techniques to recommend the best tourist spot based on the climatic condition of that place, the crowd that is present from which the users decide the areas that they need to see, visit, and for making online bookings towards accommodation and travel. 


\section{REFERENCES}

1. Ernest E.Onuiria, Henry C, Omorojeb, Chukwudi G.Ntimac, and Omotunde Ayokunie A. Intelligent tourism management system, The American Scientific Research Journal for Engineering, Technology, and Sciences, vol. 18, no. 1, pp 304-315, 2016.

2. Mr.A.Syed Mustafa, T.Gowri, M.Aishwarya, and D.Anusha Ganapathy. "Detecting Fraud Applications Using Ensemble Learning," International Journal of Future Innovative Science and Engineering Research, vol. 3, no.1, pp. 52-59, 2017.

3. Shiyu Wang and Liwei Chen. "Application of travel management system based on route inquiry," International Journal of Smart Home, DOI: 10.14257/ijsh.2015.9.6.15 June 2015. https://doi.org/10.14257/ijsh.2015.9.6.15

4. Tirth Shah, Sonal rami, and Ayesha sheikh. "Intelligent Tourist Information System," International Journal of Computer Applications, vol. 175, no.3, 2017.

https://doi.org/10.5120/ijca2017914444

5. Abdulhamid Shafii Muhammad, and Gana Usman. "Destination information management system for tourists," GESJ: Computer Science and Telecommunications, no. 6, 2010.

6. Hala Almaimoni, Njoud Altuwaijri, Fatima Asiry, Safa Aldossary, Mutasem Alsmadi, Ibrahim Al-Marashdeh, Usama A Badawi, Muneerah Alshabanah, and Daniah Alrajhi. "Developing and Implementing WEB-based Online Destination Information Management System for Tourism," International Journal of Applied Engineering Research, vol. 13, no.10, 2018.

7. W.B.Claster and Philip Pardo. "Tourism, travel, and tweets: Algorithmic text analysis methodologies in tourism," Middle East J. Management, vol. 1, no. 1, 2013.

https://doi.org/10.1504/MEJM.2013.054071

8. Joan Borràs, Antonio Moreno, and Aida Valls. "Intelligent tourism recommender systems," Elsevier Expert Systems with Applications, June 2014.

9. Damianos Gavalas a, Charalampos Konstantopoulos, and Konstantinos Mastakas. "Mobile recommender systems in tourism," Journal of Network and Computer Applications, DOI: 10.1016/j.jnca.2013.04.006, pp 319-333, 2013.

10.Fang Meng, Yodmanee Tepanon, and Muzaffer ysa. "Measuring Tourist Satisfaction by Attribute and Motivation," Journal of Vacation Marketing, DOI.org/10.1177/1356766707084218, 2008.

11. Chao Zeng, Tetsuya Nakatoh, and Sachio Hirokawa. "Text mining of tourism preference in a multilingual site." IEEE Transaction on Electrical and Electronics Engineering, DOI.org/10.1002/tee.22841, 2018.
12. R.Vasanthi, R.Jayavadivel, K.Prasadh, J.Vellingiri, G.A kilarasu, S.Sudhakar, P.M.Balasubramaniam, A novel user interaction middleware component system for ubiquitous soft computing environment by using fuzzy agent computing system, Journal of Ambient Intelligence and Humanized Computing (2020), Springer, doi.org/10.1007/s12652-020-01893-4.

13. Jagadeesh Gopal, Vellingiri J, Gitanjali J, Arivuselvan K, Sudhakar S, An Improved Trusted On-Demand Multicast Routing with QoS for Wireless Networks, International Journal of Advanced Trends in Computer Science and Engineering, Vol.9, No.1, Feb. 2020,pp:261-265,https://doi.org/10.30534/ijatcse/2020/ 39912020.

14. Satheesh N, Sudha D, Suganthi D, Sudhakar S, Dhanaraj S, Sriram VP, Priya V, "Certain improvements to Location aided packet marking and DDoS attacks in internet," Journal of Engineering Science and Technology, Vol. 15, No. 1 (2020), pp: 94 - 107, School of Engineering, Taylor's University.

15. Sathiya Kumar C, Priya V, Sriram V P, Sankar Ganesh K, Murugan G, Devi Mani, Sudhakar S, “An Efficient Algorithm for Quantum Key distribution with Secure Communication, Journal of Engineering Science and Technology, Vol. 15, No. 1 (2020), pp:77-93, School of Engineering, Taylor's University.

16. S.Sudhakar, N.Satheesh, S.Balu, Amireddy Srinish Reddy, G.Murugan, 2019, “Optimizing Joins in a Map-Reduce for Data Storage and Retrieval Performance Analysis of Query Processing in HDFS for Big Data," International Journal of Advanced Trends in Computer Science and Engineering, (IJATCSE), Vol.8, No.5, pp:2062-2067, DOI:10.30534/ijatcse/2019/33852019.

17. Sudhakar Sengan \& Chenthur Pandian S, 2016, 'Hybrid Cluster-based Geographical Routing Protocol to Mitigate Malicious Nodes in Mobile Ad Hoc Network, International Journal of Ad Hoc and Ubiquitous Computing, ISSN online: 1743-8233; ISSN print: 1743-8225, Vol.21, No.4, pp:224-236.

https://doi.org/10.1504/IJAHUC.2016.076358

18. Sudhakar Sengan, Chenthur Pandian S, 2015, “Analysis of attribute aided data aggregation through dynamic routing in wireless sensor networks," Journal of Engineering Science and Technology, School of Engineering, Taylor's University, Vol. 10, No.11 (2015) 1465-1476ISSN:1823-4690.

19. A.U. Priyadarshni, Dr.S.Sudhakar, 2015," Cluster Based Certificate Revocation by Cluster Head in Mobile Ad-Hoc Network," International Journal of Applied Engineering Research, ISSN 0973-4562 Vol. 10 No.20, pp:16014-16018.

20. Sudhakar Sengan \& Chenthur Pandian S, 2013,'Trustworthy Position-Based Routing to Mitigate against the Malicious Attacks to Signifies Secured Data Packet using Geographic Routing 
Protocol in MANET, WSEAS Transactions on Communications, vol.12, no.11, pp. 584-2013.

21. Sudhakar Sengan \& Chenthur Pandian S, 2013, 'A Trust and Co-Operative Nodes with Affects of Malicious Attacks and Measure the Performance Degradation on Geographic Aided Routing in Mobile Ad Hoc Network,' Life Science Journal, Vol. 10, No. 4s, pp. 158-163, 2013.

22. Sudhakar Sengan \& Chenthur Pandian S, 2012, 'An Efficient Agent-Based Intrusion Detection System for Detecting Malicious Nodes in MANET Routing,' International Review on Computers and Software (I.RE.CO.S.), Vol. 7, No. 6, pp. 3037-304.

23. Sudhakar Sengan \& Chenthur Pandian S, 2012, 'Secure Packet Encryption and Key Exchange System in Mobile Ad hoc Network,' Journal of Computer Science, No. 6, pp. 908-912.

https://doi.org/10.3844/jcssp.2012.908.912

24. Sudhakar Sengan \& Chenthur Pandian S, 2012. 'Authorized Node Detection and Accuracy in Position-Based Information for MANET,' European Journal of Scientific Research, Vol. 70, No. 2, pp. 253-265.

25. S.Sudhakar, V.Vijayakumar, C.SathiyaKumar, V.Priya, LogeshRavi, V.Subramaniyaswamy, Unmanned Aerial Vehicle (UAV) based Forest Fire Detection and monitoring for reducing false alarms in forest-fires, Elsevier- Computer Communications 149 (2020) 1-16, https://doi.org/10.1016/j.comcom.2019.10.007 